\title{
CXCL9 regulates acetaminophen-induced liver injury via CXCR3
}

\author{
XI SONG ${ }^{1}$, YUYING SHEN $^{1}$, YIQUN LAO $^{2}$, ZHEN TAO $^{3}$, JUAN ZENG $^{2}$, JIHUI WANG $^{1}$ and HUILING WU \\ Departments of ${ }^{1}$ General Practice, ${ }^{2}$ Infection Management and ${ }^{3}$ Infectious Diseases, \\ Nanjing First Hospital, Nanjing Medical University, Nanjing, Jiangsu 210006, P.R. China
}

Received June 5, 2018; Accepted September 23, 2019

DOI: $10.3892 /$ etm.2019.8122

\begin{abstract}
Drug-induced liver injury has become a serious public health problem. Although the mechanism of acetaminophen (APAP)-induced liver injury has been studied for decades it has not been fully elucidated. In-depth study into the mechanisms underlying APAP-induced liver injury may provide useful information for more effective prevention and treatment. In the present study, the role of C-X-C motif chemokine ligand-9 (CXCL9) in APAP-induced liver injury was investigated thus providing a novel direction for the prevention and treatment of drug hepatitis. A total of 20 fasting male patients ingested APAP tablets at Nanjing First Hospital. In addition, wild type (WT) mice were treated with $250 \mathrm{mg} / \mathrm{kg}$ APAP or isodose PBS for 1, 3, 6 and $12 \mathrm{~h}$, respectively. Results from reverse-transcription-quantitative polymerase chain reaction analyses demonstrated that CXCL9 mRNA levels were increased in the blood of patients who took APAP in a fasting state and in the livers of APAP-treated WT mice, compared with their respective controls. Hepatocyte apoptosis in the liver tissue of APAP-treated mice decreased following administration of a CXCL9 neutralizing antibody. Caspase-3, caspase- 8 and phosphorylated-AKT (S437) were activated in primary hepatocytes isolated from WT mice following CXCL9 treatment. However, no significant differences in expression of caspase-3, caspase- 8 and p-AKT (S437) were detected in hepatocytes isolated from $\mathrm{C}-\mathrm{X}-\mathrm{C}$ motif chemokine receptor 3 (CXCR3) $^{-/-}$mice following CXCL9 treatment. After CXCL9 administration, WT mice exhibited higher serum levels of aspartate transaminase and increased caspase- 3 and caspase- 8 activity in liver tissue compared with controls. The same trends were not observed in CXCR $3^{-/}$mice. In conclusion, CXCL9 regulated APAP-induced liver injury through stimulation of hepatocyte apoptosis via binding to CXCR3. These findings provide a novel prevention and treatment strategy for DILI.
\end{abstract}

Correspondence to: Dr Huiling Wu, Department of General Practice, Nanjing First Hospital, Nanjing Medical University, 68 Changle Road, Nanjing, Jiangsu 210006, P.R. China

E-mail: wuhuiling08@126.com

Key words: acetaminophen, drug-induced liver injury, C-X-C motif chemokine ligand 9, $\mathrm{C}-\mathrm{X}-\mathrm{C}$ motif chemokine receptor 3

\section{Introduction}

Drug-induced liver injury (DILI) is a common side effect of drug administration and is also one of the leading causes of acute liver failure and liver transplantation in Western countries (1). DILI has a significant role in failure of numerous new drug developments and withdrawals from the market $(2,3)$.

Acetaminophen (APAP) is an acetanilide antipyretic analgesic, which is frequently utilized as an over-the-counter drug due to its safety and reliability. APAP has been used as an adjunct therapy for acute and chronic hepatitis in China for several decades (4). However, APAP overdose is one of the leading causes of DILI globally accounting for $>50 \%$ of cases (5). It has proven difficult to elucidate the specific pathogenesis of DILI due to the differing severity of symptoms between patients resulting from individual differences (6).

A small amount of APAP in the liver produces biologically active N-acetyl-p-benzoquinone imine (NAPQI), which is detoxified via binding to glutathione (GSH). APAP overdose results in NAPQI accumulation in the liver, thereby over-consuming GSH (7). GSH deficiency inhibits the function of peroxide inactivation leading to further accumulation in the body (8). It is reported that the subsequent inflammatory response cascade serves an essential role in DILI (9). Among multiple pathological changes in DILI, hepatocyte apoptosis is considered to be the leading cause of APAP-induced liver injury $(10,11)$. Therefore, investigation into the pathogenesis of APAP-induced liver injury is of great significance to improve clinical outcomes of affected patients.

Chemokines are capable of inducing immune cell migration to inflammatory lesions and they directly affect the function of intrinsic cells (12). C-X-C motif chemokine receptor 3 (CXCR3) and its ligands, C-X-C motif chemokine ligand (CXCL)-9, CXCL10 and CXCL11 have been reported to be involved in various pathological processes, such as tumor development, immunity and inflammation $(13,14)$. Chemokines are difficult to detect in non-lymphoid tissues under physiological conditions, however they are released at high levels during inflammation, infection or injury (15). CXCL9 and its receptors have been widely reported to regulate tumor invasion, metastasis, leukocyte invasion and T cell activation (16). The specific role of CXCL9 in cell apoptosis remains unclear. In the present study, it was hypothesized that CXCL9 could promote hepatocyte apoptosis via binding to CXCR3, thereby aggravating APAP-induced liver injury. 


\section{Materials and methods}

Subjects. A total of 20 healthy men aged 24-66 (37.2 \pm 12.0$)$ ingested APAP $(500 \mathrm{mg}$ ) after fasting for $24 \mathrm{~h}$; as a control a further 20 healthy male volunteers, aged 18-63 (37.8 \pm 10.7$)$, who had fasted for 24 but not ingested APAP were enrolled in this study. $5 \mathrm{ml}$ of blood was acquired from the peripheral vein of each patient and the blood samples were then centrifuged at $2,500 \mathrm{x} \mathrm{g}$ at $4^{\circ} \mathrm{C}$ for $5 \mathrm{~min}$ to obtain serum. None of the subjects had suffered from viral hepatitis or other autoimmune diseases, and there was no statistically significant difference in ages between the two groups.

Experimental animals. CXCR $3^{--}$mice used in the present study were obtained from the Jackson Laboratory (Bar Harbor, ME, USA). A total of 1008 week-old male wild type (WT) mice and $50 \mathrm{CXCR}^{-/}$mice weighing between $18-22 \mathrm{~g}$, were used for experiments, with 6-8 mice in each group. All mice were housed individually in a specific pathogen-free level animal experimental center with free access to water and food.

Animal model construction. The 8 week-old male WT and $\mathrm{CXCR}^{-/-}$mice were intraperitoneally injected with $250 \mathrm{mg} / \mathrm{kg}$ APAP (5). Immediately after this, the experimental group mice were further injected with $100 \mu \mathrm{g}$ (in $100 \mu \mathrm{l}$ volume) CXCL9 neutralizing antibody (cat no 50155-R277; Sino Biological Inc., Beijing, China) or $5 \mu \mathrm{g}$ (in $100 \mu 1$ volume) recombinant CXCL9 (cat no 50155-MNAE; Sino Biological Inc.). Mice in the control group were injected with an equivalent dose of control IgG or saline. A total of $0.1 \mathrm{ml}$ blood was collected from the tail vein of each mouse before the mice were sacrificed using 5\% isoflurane followed by cervical dislocation. Mice were sacrificed and liver tissues were harvested after $8(n=3)$ or $24(n=3)$ h. Blood samples were centrifuged at $4,500 \mathrm{x} \mathrm{g}$ at $4^{\circ} \mathrm{C}$ for $5 \mathrm{~min}$ to obtain serum.

Biochemical and pathological analyses. Serum samples from APAP-treated WT and CXCR $3^{-/}$mice were collected for the analysis of alanine aminotransferase (ALT) and aspartate aminotransferase (AST) levels. Biochemical analyzer (model AU480; Beckman Coulter, Inc.) was used for ALT and AST colorimetric detection. Caspase-3 expression in liver tissues was determined using a caspase-3 detection kit (Beyotime Institute of Biotechnology; cat. no. C1168S) according to the instructions of the manufacturer.

Transferase-mediated deoxyuridine triphosphate-biotin nick end labeling (TUNEL) staining. Mice were sacrificed, then livers were dissected. After overnight incubation in $4 \%$ paraformaldehyde, livers were dehydrated and embedded in paraffin. Sections of $5 \mu \mathrm{m}$ thickness were cut and mounted on poly-lysine-coated slides, and hepatocyte apoptosis was detected using a TUNEL assay (Beyotime Institute of Biotechnology) according to the instructions of the manufacturer.

Hematoxylin and eosin $(H \& E)$ staining. Mice were sacrificed and their livers were dissected. After overnight incubation at room temperature in $4 \%$ paraformaldehyde, livers were dehydrated and embedded in paraffin. Sections of $5 \mu \mathrm{m}$ thickness were cut and mounted on poly-lysine-coated slides (10 slides per tissue). After H\&E staining (hematoxylin, $5 \mathrm{~min}$ at room temperature and eosin, $3 \mathrm{~min}$ at $20^{\circ} \mathrm{C}$ ) sections were observed (5 views per slide) with a light microscope (magnification $\mathrm{x} 400$ ).

Reverse transcription-quantitative polymerase chain reaction $(R T-q P C R)$. Total RNA was extracted from cells using the TRIzol ${ }^{\circledR}$ method (Invitrogen; Thermo Fisher Scientific, Inc., Waltham, MA, USA) then quantified. cDNA was synthesized using the Prime Script ${ }^{\mathrm{TM}}$ RT-PCR Kit (Takara Bio, Inc., Otsu, Japan) according to the instructions of the manufacturer. A total of $2 \mu \mathrm{l}$ cDNA was used for each reaction, amplified by real-time PCR using SYBR ${ }^{\circledR}$ Premix Ex Taq ${ }^{\mathrm{TM}}$ II (Takara Bio, Inc.). Relative mRNA expression was analyzed by qPCR using the IQ ${ }^{\mathrm{TM}} 5$ System (Bio-Rad Laboratories, Inc., Hercules, CA, USA). Primers were synthesized by Shanghai ShengGong Co., Ltd. (Shanghai, China). Primer sequences were as follows: CXCL9, forward 5'-CCTAGTGATAAGGAATGCACG ATG-3' and reverse 5'-CTAGGCAGGTTTGATCTCCGT TC-3'; and GAPDH, forward 5'-TGAAGGTCGGAGTCA ACGGATT-3' and reverse 5'-CCTGGAAGATGGTGATGG GATT-3'. The $2^{-\Delta \Delta C a}$ method was used to calculate the relative mRNA expression (17). The thermocycling conditions were as follows: Initial denaturation at $95^{\circ} \mathrm{C}$ for $5 \mathrm{~min}$, followed by 40 cycles of $95^{\circ} \mathrm{C}$ for $30 \mathrm{sec}, 95^{\circ} \mathrm{C}$ for $5 \mathrm{sec}$ and $60^{\circ} \mathrm{C}$ for $31 \mathrm{sec}$, before a final extension at $94^{\circ} \mathrm{C}$ for $15 \mathrm{sec}$. Each experiment was repeated in triplicate.

Western blot analysis. Cells were lysed with RIPA buffer (Beyotime Institute of Biotechnology) containing protease inhibitors. Protein concentration was quantified using bicinchoninic acid protein assay (Pierce; Thermo Fisher Scientific, Inc.). Protein samples $(10 \mu \mathrm{g})$ were separated on $12 \%$ SDS-PAGE gel and transferred to polyvinylidene fluoride membranes (Roche, Basel, Switzerland). Membranes were incubated in $5 \%$ non-fat milk at $25^{\circ} \mathrm{C}$ for $1 \mathrm{~h}$ to block non-specific binding. Membranes were then membranes incubated overnight at $4^{\circ} \mathrm{C}$ with primary antibodies targeting phosphorylated (p-) Akt (cat. no. ab81283; 1:500; Abcam, Cambridge, MA, USA), total Akt (cat. no. ab18785; 1:500; Abcam), and $\beta$-actin (cat. no. ab8226; 1:500; Abcam). Membranes were washed three times with PBS, then samples were incubated with anti-rabbit or anti-mouse secondary antibodies purchased from Beyotime Institute of Biotechnology (cat. no. A0208/A0216; 1:1,000). Bands were visualized using enhanced chemiluminescence substrate (Thermo Fisher Scientific Inc.). Quantity One (version 4.0; Bio-Rad Laboratories, Inc.) was used for densitometric analysis.

ELISA. Human serum samples were collected and CXCL9 levels were determined, in accordance with ELISA kit instructions (cat. no. ab119588; Abcam). The absorbance of each sample was detected at wavelengths 562 and $450 \mathrm{~nm}$.

Isolation and culture of primary hepatocytes. Isolation and culture of primary hepatocytes were performed as previously described (18). In brief, WT and CXCR $3^{-/}$mice were sacrificed for access to the liver. Liver tissues were digested with digestive buffer containing collagenase, ground, and then 

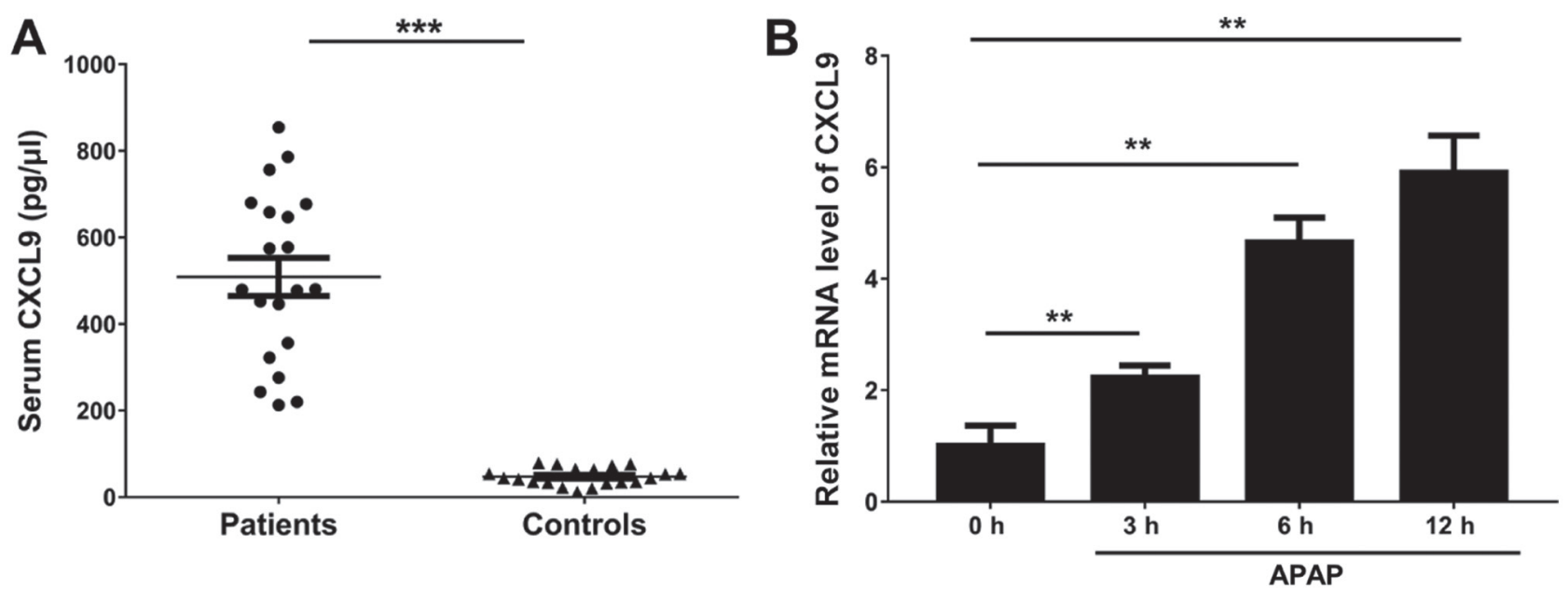

Figure 1. CXCL9 participates in the pathological process of APAP-induced liver injury. (A) Serum levels of CXCL9 were significantly higher in patients who took APAP compared with controls. (B) Compared with mice injected with PBS, those injected with APAP presented increased mRNA levels of CXCL9 lasting up to $12 \mathrm{~h}$. ${ }^{* *} \mathrm{P}<0.01$ and ${ }^{* * * *} \mathrm{P}<0.001$, with comparisons indicated by lines. CXCL9, C-X-C motif chemokine ligand-9; APAP, acetaminophen.

filtered for preparation of single cell suspension. After gradient centrifugation and lysis with red blood cell lysis buffer, mouse primary hepatocytes were obtained. Cells were cultured in William's E medium (Gibco; Thermo Fisher Scientific, Inc.) for $14 \mathrm{~h}$, serum-starved for $3 \mathrm{~h}$, and then treated with $100 \mathrm{ng} / \mathrm{ml} \mathrm{CXCL9}$. Protein samples were collected at $5 \mathrm{~min}$, $20 \mathrm{~min}, 1,4$ or $8 \mathrm{~h}$ post-treatment.

Statistical analysis. Graphpad Prism (v6.0) software (GraphPad Software, Inc., La Jolla, CA, USA) was used for statistical analysis. Data are presented as the mean \pm the standard deviation. Differences amongst groups were compared using one-way analysis of variance, followed by Bonferroni correction. $\mathrm{P}<0.05$ was considered to indicate significant difference.

\section{Results}

CXCL9 participates in the pathological process of APAP-induced liver injury. Serum levels of CXCL9 were determined in 20 male patients who took APAP tablets in a fasting state and in 20 fasted male controls. The results demonstrated that serum levels of CXCL9 were significantly higher in patients who took APAP compared with the control group (Fig. 1A).

For the animal experiments, male WT mice were randomly assigned into one of two groups, receiving either intraperitoneal injection of APAP or PBS. The mRNA levels of CXCL9 in liver tissues were detected at 1,3, 6 and $12 \mathrm{~h}$ following injection. Compared with mice injected with PBS, those injected with APAP presented significantly increased mRNA levels of CXCL9, even up to $12 \mathrm{~h}$ post-injection (Fig. 1B).

Neutralization of CXCL9 attenuates APAP-induced liver injury. Following intraperitoneal injection of APAP, WT mice received intraperitoneal injection of anti-CXCL9 neutralizing antibody or control immunoglobulin (IgG). Serum and liver samples were collected after 8 and 24 h. Serum levels of ALT and AST were lower in mice injected with anti-CXCL9 neutralizing antibody and $\mathrm{CXCR} 3^{-/}$mice, compared with the control group of WT mice that received APAP and IgG only (Fig. 2A and B). There were no significant differences in ALT and AST levels between mice injected with anti-CXCL9 neutralizing antibody and $\mathrm{CXCR}^{-/-}$mice. (Fig. 2A and $\mathrm{B}$ ). HE staining revealed that neutralization of CXCL9 attenuated APAP-induced liver injury and knockout of CXCR3 also alleviated APAP-induced liver injury (Fig. 2C). TUNEL assay indicated that hepatocyte apoptosis was alleviated in liver tissues from the anti-CXCL9 neutralizing antibody group and the $\mathrm{CXCR}^{-/-}$group (Fig. 2D). These results suggested that neutralization of CXCL9 or knockout of CXCR3 attenuated APAP-induced liver injury.

CXCL9 promotes hepatocyte apoptosis via the AKT pathway. To further explore the specific mechanism of CXCL9 in APAP-induced liver injury, primary hepatocytes were isolated from WT mice for subsequent experiments. Primary hepatocytes were treated with or without recombinant CXCL9 for different time periods. Activities of caspase- 3 and caspase- 8 in hepatocytes were significantly elevated following CXCL9 treatment for 4 and $8 \mathrm{~h}$ (Fig. 3A and B). The AKT pathway was activated within the first 5 min of CXCL9 treatment in a time-dependent manner, and the activation lasted for up to $8 \mathrm{~h}$ (Fig. 3C and D).

CXCL9 induces hepatocyte apoptosis via binding to CXCR3. Studies have demonstrated that CXCL9 is involved in tumor metastasis and the inflammatory response via binding to the CXCR3 receptor. Hence, it was hypothesized that CXCL9 regulates hepatocyte apoptosis through CXCR3 in APAP-induced liver injury. Primary hepatocytes from $\mathrm{CXCR}^{-/-}$mice were treated with recombinant CXCL9 or vehicle control. Activities of caspase- 3 and caspase- 8 were measured by fluorescence spectrophotometry. There was no significant difference in expression of caspase- 3 and caspase- 8 after CXCL9 stimulation for 4 and $8 \mathrm{~h}$ (Fig. 4A and B). In addition, there was no significant difference in p-AKT (S437) expression for up to $8 \mathrm{~h}$ following CXCL9 treatment, compared with control untreated CXCR3 ${ }^{-/-}$hepatocytes (Fig. 4C and D). The present 

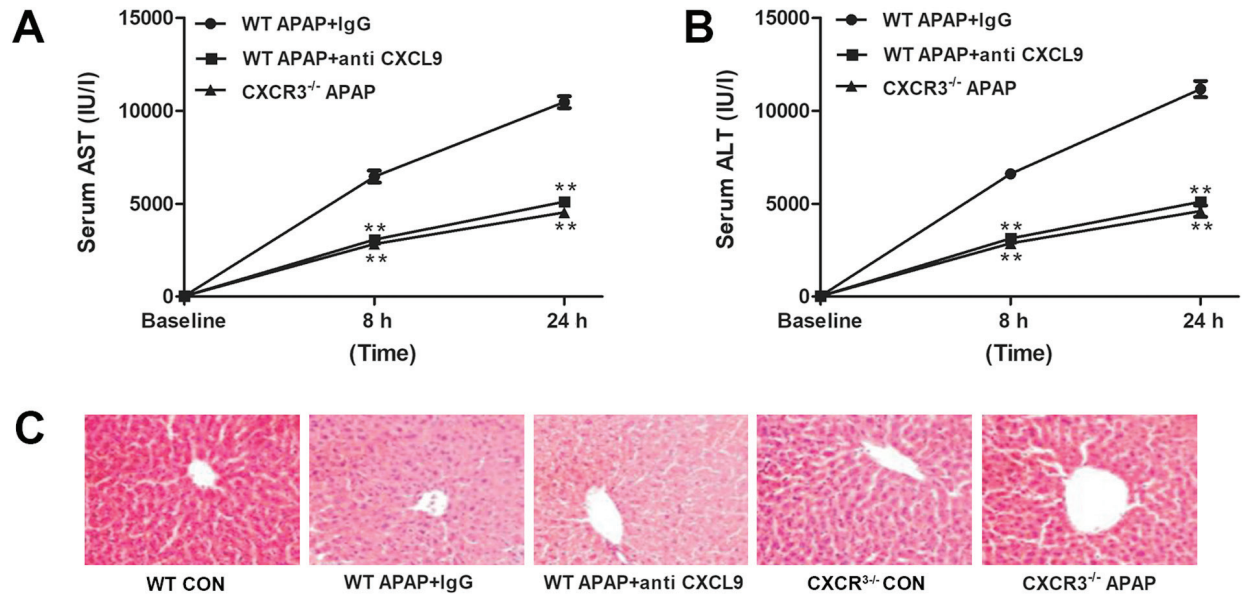

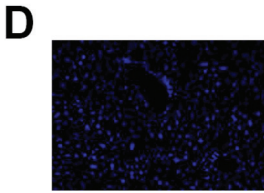

WT CON

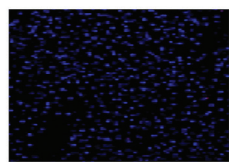

$\mathrm{CXCR}^{3+\cdot} \cdot \mathrm{CON}$

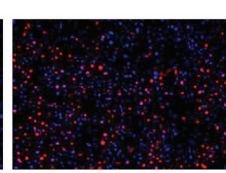

WT APAP+lgG

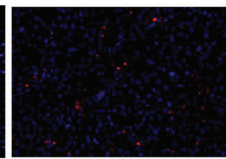

$\mathrm{CXCR}^{-1-}$ APAP

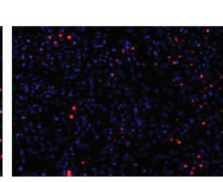

WT APAP+anti CXCL9

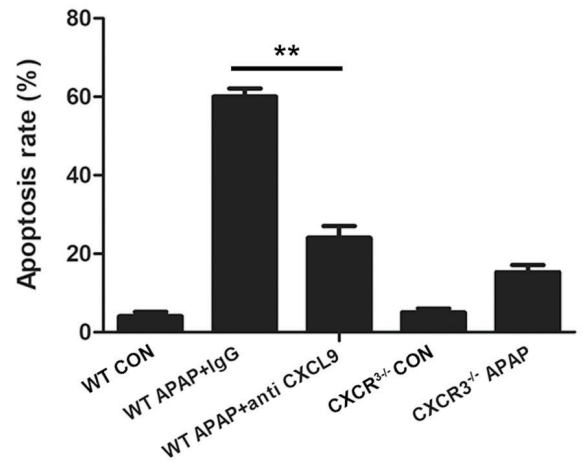

Figure 2. Neutralization of CXCL9 attenuates APAP-induced liver injury. (A) Serum levels of ALT and (B) AST were significantly lower in mice injected with anti-CXCL9 neutralizing antibody compared with controls. ${ }^{* *} \mathrm{P}<0.01$ vs. WT APAP+IgG. (C) Neutralization of CXCL9 attenuated APAP-induced liver injury, as evidenced by HE staining (magnification x200). (D) TUNEL assay indicated that hepatocyte apoptosis was alleviated in liver tissues following anti-CXCL9 neutralizing antibody administration (magnification $\mathrm{x} 200$ ). ${ }^{* *} \mathrm{P}<0.01$, with comparisons indicated by lines. CXCL9, C-X-C motif chemokine ligand-9; APAP, acetaminophen; ALT, alanine aminotransferase; AST, aspartate aminotransferase; WT, wild-type; HE, hematoxylin and eosin; TUNEL, transferase-mediated deoxyuridine triphosphate-biotin nick end labeling.

A

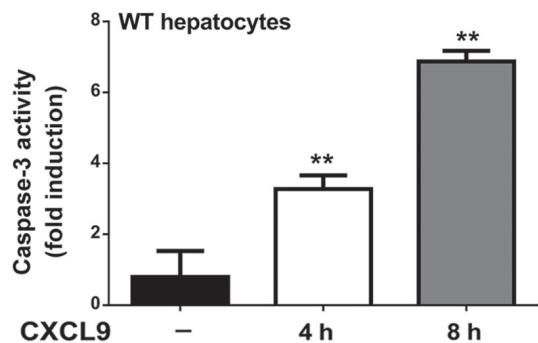

C

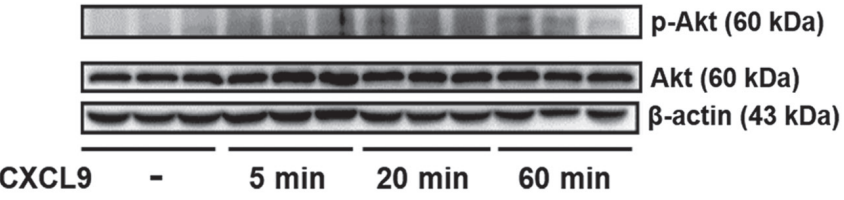

D

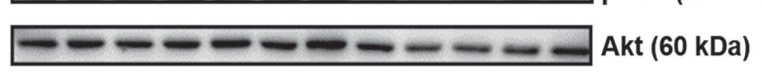

CXCL9 -

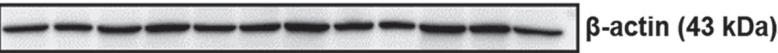

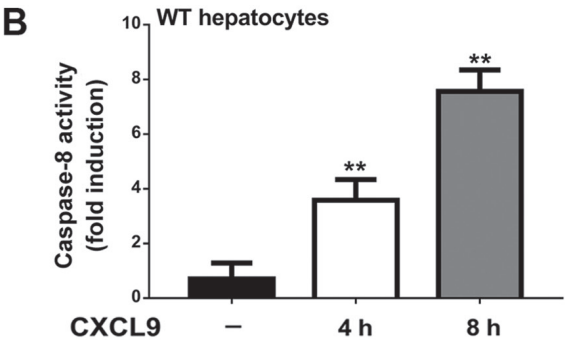
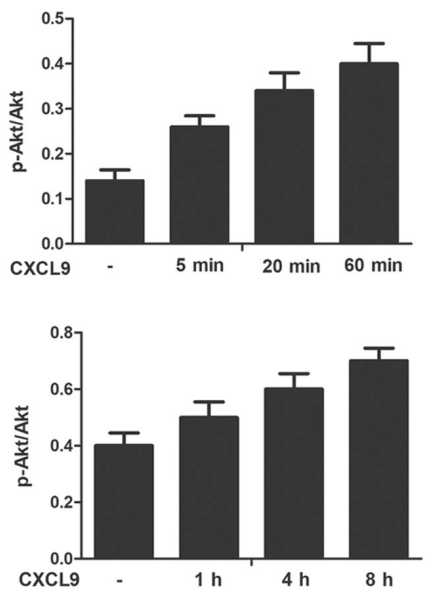

Figure 3. CXCL9 promotes hepatocyte apoptosis via the AKT pathway. (A) Activities of caspase-3 and (B) caspase-8 in primary WT hepatocytes were elevated following CXCL9 treatment for 4 and $8 \mathrm{~h}$. (C and D) The AKT pathway was activated within the first 5 min of CXCL9 treatment in a time-dependent manner, with the effect lasting up to $8 \mathrm{~h} .{ }^{* *} \mathrm{P}<0.01$ vs. untreated control. CXCL9, C-X-C motif chemokine ligand-9; AKT, protein kinase B; WT, wild type. 
A

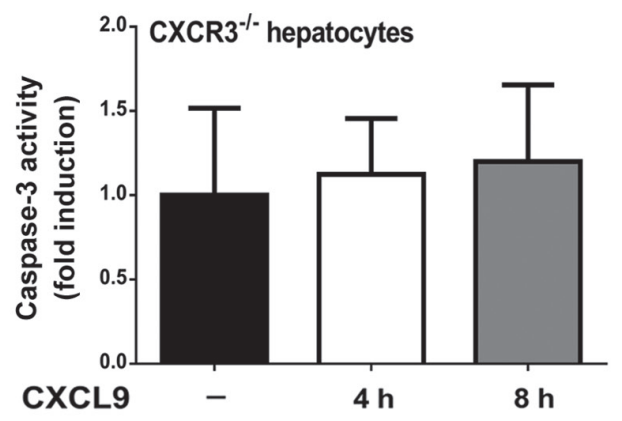

C

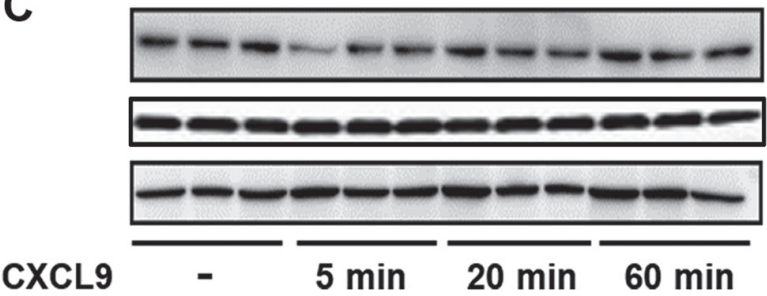

D
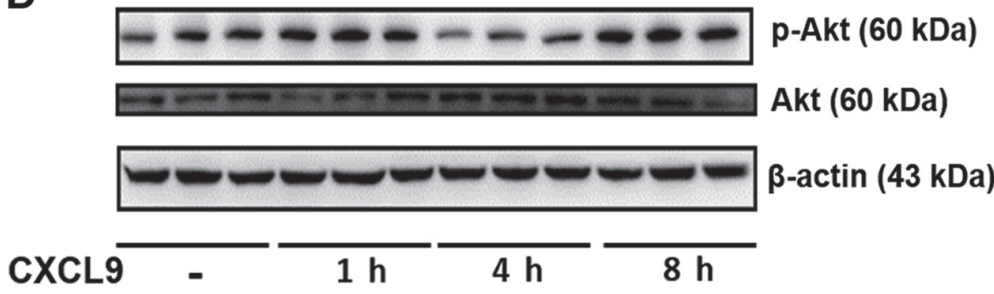

B
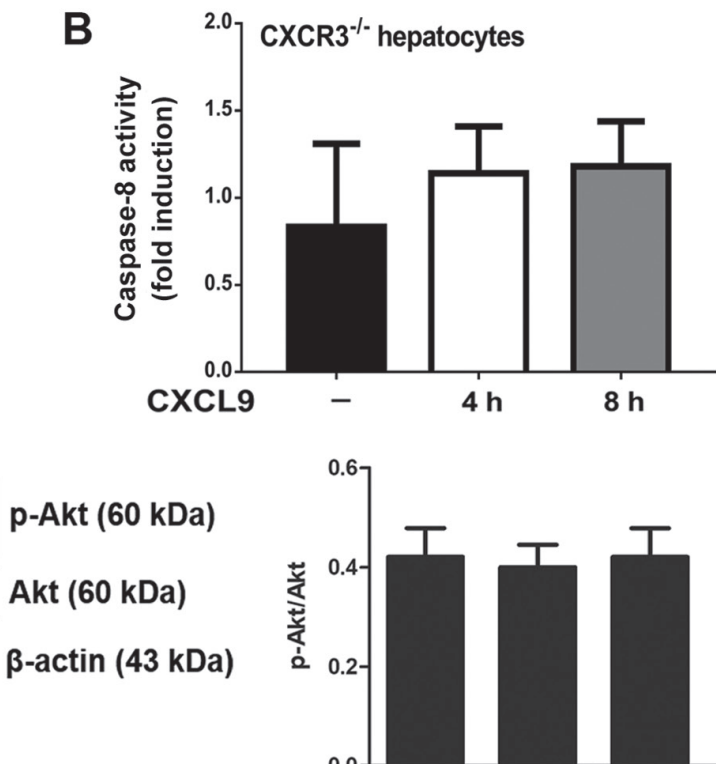

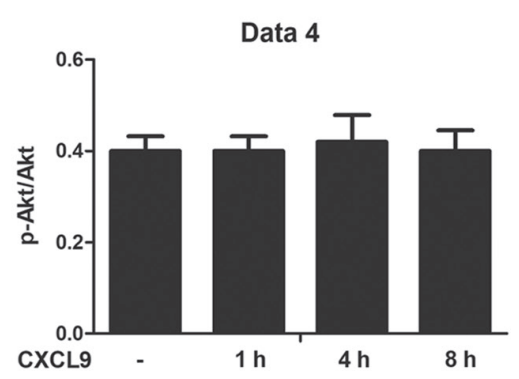

Figure 4. CXCL9 induces hepatocyte apoptosis via binding to CXCR3. (A) No significant differences in expression of caspase-3 and (B) caspase-8 were detected after CXCL9-stimulation for 4 and $8 \mathrm{~h}$ in primary hepatocytes isolated from CXCR $3^{-\%}$ mice. (C and D) There was no significant difference in AKT pathway activation after CXCL9 treatment for $8 \mathrm{~h}$. CXCL9, C-X-C motif chemokine ligand-9; CXCR3, C-X-C motif chemokine receptor 3; AKT, protein kinase B
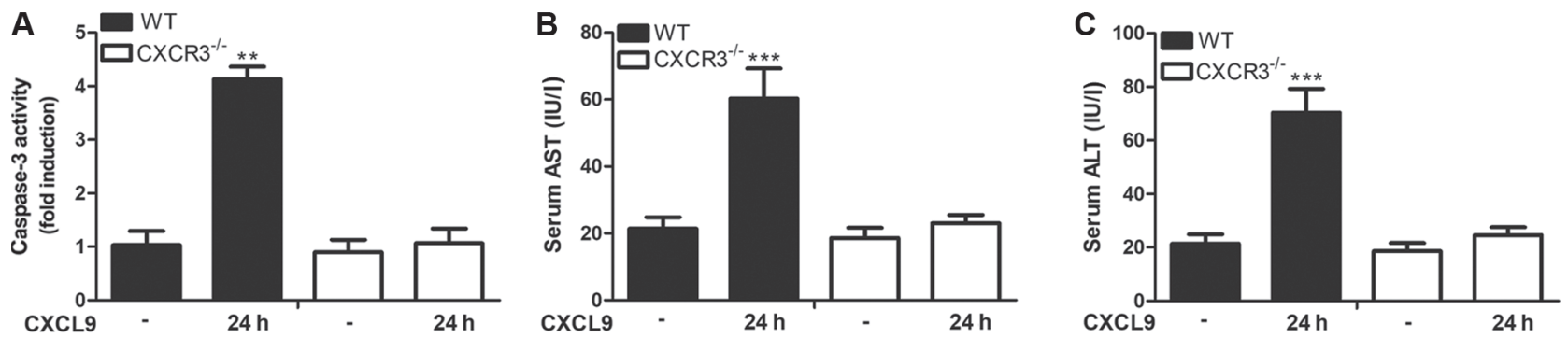

Figure 5. CXCL9 induces CXCR3-mediated apoptosis in mouse liver. (A) At $24 \mathrm{~h}$ following intraperitoneal injection of CXCL9, caspase-3 activity in the liver tissues of WT mice was significantly higher compared with the liver tissues of CXCR $3^{-/}$mice. (B) Serum levels of ALT and (C) AST were also higher in WT mice injected with CXCL9 compared with CXCR3 ${ }^{--}$mice. ${ }^{* *} \mathrm{P}<0.01,{ }^{* * *} \mathrm{P}<0.001$ vs. control. CXCL9, C-X-C motif chemokine ligand-9; CXCR3, C-X-C motif chemokine receptor 3; WT, wild type; ALT, alanine aminotransferase; AST, aspartate aminotransferase.

data demonstrated that CXCL9 exerted its function in APAP-induced liver injury via binding to CXCR3.

CXCL9 induces CXCR3-mediated apoptosis in mouse liver. Mouse experiments were further performed to confirm the roles of CXCL9 and CXCR3 in APAP-induced hepatocyte apoptosis in vivo. Intraperitoneal injections of CXCL9 were administered to both WT and $\mathrm{CXCR}^{-/-}$mice. At $24 \mathrm{~h}$ following CXCL9 administration, the caspase- 3 activity in the liver tissues of WT mice was significantly higher compared with $\mathrm{CXCR}^{-/-}$mice
(Fig. 5A). In addition, serum levels of ALT and AST were also significantly higher in WT mice compared with $\mathrm{CXCR}^{-/-}$mice, following $24 \mathrm{~h}$ of CXCL9 administration (Fig. 5B and C).

\section{Discussion}

The present study, demonstrated that APAP administration resulted in increased secretion of CXCL9, thereby stimulating hepatocyte apoptosis and leading to liver damage through binding to CXCR3 and via the AKT pathway. 
Three major steps are involved in APAP-induced liver injury. Firstly, APAP and its metabolites directly lead to the hepatocyte stress response (intrinsic pathway), activation of the immune response (extrinsic pathway) and mitochondrial dysfunction. Secondly, mitochondrial permeability transition pores (MPTs) open. Finally, MPT opening leads to apoptosis and necrosis of liver cells (19). It had been previously reported that cytokines are involved in the pathogenesis of various liver diseases, particularly in immune diseases induced by inflammation and $\mathrm{T}$ cells $(12,20)$.

The present results suggested that administration of a neutralizing CXCL9 antibody significantly reduced APAP-induced liver injury in WT mice. Hepatocyte apoptosis was increased after CXCL9-treatment in primary hepatocytes. CXCR3 is the common receptor of CXCL9 and has been well studied for many diseases $(15,16)$. The present data confirmed that interaction between CXCL9 and CXCR3 is involved in hepatocyte apoptosis induced by APAP. CXCR3 deficiency did not induce apoptosis of mouse hepatocytes both in vitro and in vivo.

The downstream mechanism of CXCL9 binding to CXCR3 has not been clearly elucidated. The present study detected expression of apoptosis and transcription-related signaling pathways following CXCL9 stimulation. Surprisingly, AKT was phosphorylated after APAP induction indicating that CXCL9 regulates APAP-induced liver injury via the AKT pathway.

Inflammatory factors and macrophages are considered to be associated with APAP-induced liver injury (21-24). The present study hypothesized that hepatocyte apoptosis was the most important factor for APAP-induced liver injury and subsequent progression to liver cirrhosis which is consistent with previous studies (10-12). Strict control of hepatocyte apoptosis contributes to inhibition of inflammatory factors in necrotic tissues.

It is of great significance to understand the pathogenesis of DILI in order to prevent and treat clinical DILI. It has been suggested that DILI is regulated by various aspects, such as drug metabolism, mitochondrial function impairment, immune response, signal transduction, genetic and environmental factors. The present study determined that CXCL9 and CXCR3 exerted key roles in hepatocyte apoptosis induced by APAP. Taken together, these findings suggest that CXCL9 may be a potential therapeutic intervention target for acute hepatitis and liver failure, and could provide a novel prevention and treatment strategy for DILI.

\section{Acknowledgements}

Not applicable.

\section{Funding}

No funding was received.

\section{Availability of data and materials}

All data generated or analyzed during this study are included in this published article.

\section{Authors' contributions}

XS and HW designed the study and performed the experiments, YS, YL and ZT established the animal models, YS, JZ and JW collected the data, XS and YS analyzed the data, XS and HW prepared the manuscript. All authors read and approved the final manuscript.

\section{Ethics approval and consent to participate}

This study was approved by the Animal Ethics Committee of Nanjing Medical University Animal Center, and was approved by the ethics committee of Nanjing First Hospital. Signed written informed consents were obtained from the patients and/or their guardians.

\section{Patient consent for publication}

Not applicable.

\section{Competing interests}

The authors declare that they have no competing interests.

\section{References}

1. Larson AM, Polson J, Fontana RJ, Davern TJ, Lalani E, Hynan LS, Reisch JS, Schiodt FV, Ostapowicz G, Shakil AO, et al: Acetaminophen-induced acute liver failure: Results of a united states multicenter, prospective study. Hepatology 42: 1364-1372, 2005.

2. Senior JR: Drug hepatotoxicity from a regulatory perspective. Clin Liver Dis 11: 507-524, 2007.

3. Atienzar FA, Blomme EA, Chen M, Hewitt P, Kenna JG, Labbe G, Moulin F, Pognan F, Roth AB, Suter-Dick L, et al: Key challenges and opportunities associated with the use of in vitro models to detect human DILI: Integrated risk assessment and mitigation plans. Biomed Res Int 2016: 9737920, 2016.

4. Zhou M, Wang M, Zhong RF, Liao XM, Deng LL, Xu GB, He X, Li J, Li YJ, Liu T, et al: Discovery and structure-activity relationship of auriculatone: A potent hepatoprotective agent against acetaminophen-induced liver injury. Bioorg Med Chem Lett 27: 3636-3642, 2017

5. Xu XY, Hu JN, Liu Z, Zhang R, He YF, Hou W, Wang ZQ, Yang G and Li W: Saponins (Ginsenosides) from the leaves of Panax quinquefolius ameliorated acetaminophen-induced hepatotoxicity in mice. J Agric Food Chem 65: 3684-3692, 2017.

6. Kim M, Yun JW, Shin K, Cho Y, Yang M, Nam KT and Lim KM: Expression levels of GABA-A receptor subunit alpha 3, gabra3 and lipoprotein lipase, $1 \mathrm{pl}$ are associated with the susceptibility to acetaminophen-induced hepatotoxicity. Biomol Ther (Seoul) 25: 112-121, 2017.

7. Larson AM: Acetaminophen hepatotoxicity. Clin Liver Dis 11: 525-548, 2007.

8. James LP, Mayeux PR and Hinson JA: Acetaminophen-induced hepatotoxicity. Drug Metab Dispos 31: 1499-1506, 2003.

9. Granger DN, Vowinkel T and Petnehazy T: Modulation of the inflammatory response in cardiovascular disease. Hypertension 43: 924-931, 2004.

10. Wu H, Zhang G, Huang L, Pang H, Zhang N, Chen Y and Wang G: Hepatoprotective effect of polyphenol-enriched fraction from folium microcos on oxidative stress and apoptosis in acetaminophen-induced liver injury in mice. Oxid Med Cell Longev 2017: 3631565, 2017.

11. Yan XT, Sun YS, Ren S, Zhao LC, Liu WC, Chen C, Wang Z and Li W: Dietary $\alpha$-mangostin provides protective effects against acetaminophen-induced hepatotoxicity in mice via Akt/mTOR-mediated inhibition of autophagy and apoptosis. Int J Mol Sci 19: E1335, 2018. 
12. Sahin H, Borkham-Kamphorst E, Do ON, Berres ML, Kaldenbach M, Schmitz P, Weiskirchen R, Liedtke C, Streetz KL, Maedler K, et al: Proapoptotic effects of the chemokine, CXCL 10 are mediated by the noncognate receptor TLR4 in hepatocytes. Hepatology 57: 797-805, 2013.

13. Han X, Wu Z, Di J, Pan Y,Zhang H, Du Y, Cheng Z, Jin Z, Wang Z, Zheng Q, et al: CXCL9 attenuated chemotherapy-induced intestinal mucositis by inhibiting proliferation and reducing apoptosis. Biomed Pharmacother 65: 547-554, 2011.

14. Tokunaga R, Zhang W, Naseem M, Puccini A, Berger MD, Soni S, McSkane M, Baba H and Lenz HJ: CXCL9, CXCL10, CXCL11/CXCR3 axis for immune activation-a target for novel cancer therapy. Cancer Treat Rev 63: 40-47, 2018.

15. Muller M, Carter S, Hofer MJ and Campbell IL: Review: The chemokine receptor CXCR3 and its ligands CXCL9, CXCL10 and CXCL11 in neuroimmunity-a tale of conflict and conundrum. Neuropathol Appl Neurobiol 36: 368-387, 2010 .

16. Liu W, Ren HY, Dong YJ, Wang LH, Yin Y, Li Y, Qiu ZX, Cen XN and Shi YJ: Bortezomib regulates the chemotactic characteristics of $\mathrm{T}$ cells through downregulation of CXCR3/CXCL9 expression and induction of apoptosis. Int J Hematol 96: 764-772, 2012.

17. Livak KJ and Schmittgen TD: Analysis of relative gene expression data using real-time quantitative PCR and the 2(-Delta Delta C(T)) method. Methods 25: 402-408, 2001.

18. Cassim S, Raymond VA, Lapierre P and Bilodeau M: From in vivo to in vitro: Major metabolic alterations take place in hepatocytes during and following isolation. PLoS One 12: e190366, 2017.
19. Henderson NC, Pollock KJ, Frew J, Mackinnon AC, Flavell RA, Davis RJ, Sethi T and Simpson KJ: Critical role of c-jun (NH2) terminal kinase in paracetamol-induced acute liver failure. Gut 56: 982-990, 2007.

20. Yu J, Wu H, Liu ZY, Zhu Q, Shan C and Zhang KQ: Advanced glycation end products induce the apoptosis of and inflammation in mouse podocytes through CXCL9-mediated JAK2/STAT3 pathway activation. Int J Mol Med 40: 1185-1193, 2017.

21. Nakamoto N, Ebinuma H, Kanai T, Chu PS, Ono Y, Mikami Y, Ojiro K, Lipp M, Love PE, Saito H and Hibi T: CCR9+ macrophages are required for acute liver inflammation in mouse models of hepatitis. Gastroenterology 142: 366-376, 2012.

22. Schumann J, Wolf D, Pahl A, Brune K, Papadopoulos T, van Rooijen N and Tiegs G: Importance of kupffer cells for T-cell-dependent liver injury in mice. Am J Pathol 157: 1671-1683, 2000.

23. Nakashima H, Kinoshita M, Nakashima M, Habu Y, Shono S, Uchida T, Shinomiya N and Seki S: Superoxide produced by Kupffer cells is an essential effector in concanavalin A-induced hepatitis in mice. Hepatology 48: 1979-1988, 2008.

24. Bonder CS, Ajuebor MN, Zbytnuik LD, Kubes P and Swain MG: Essential role for neutrophil recruitment to the liver in concanavalin A-induced hepatitis. J Immunol 172: 45-53, 2004.

(i) $(2)$ This work is licensed under a Creative Common Attribution-NonCommercial-NoDerivatives 4.0 International (CC BY-NC-ND 4.0) License. 